\title{
Insulin-driven translational capacity is impaired in primary fibroblasts of Prader Willi
}

\author{
Cristiana Meneghello, Daniela Segat, Elisabetta Fortunati* \\ "Mauro Baschirotto" Institute for Rare Diseases - B.I.R.D., Vicenza. Italy.
}

\begin{abstract}
Summary Prader-Willi (PW) syndrome is a rare genetic disorder characterized by hypothalamicpituitary abnormalities and severe hypotonia, hyperphagia, behavioural and psychiatric problems. Absence of satiety leads to severe obesity and frequently to diabetes. Furthermore, adult patients suffer from a severe loss of muscle mass, which severely impacts their quality of life. The mechanisms underlying alterations in muscle growth in PW remain to be clarified. In this study we explored the hypothesis that, in PW cells, alterations of protein synthesis are determined by dysfunctions in the promotion of cell growth. In order to study the molecular changes leading to dysfunction in protein translation, primary fibroblasts derived from four PW patients and five control subjects were used to study the insulin-mediated signaling pathway implicated in the control of protein synthesis by immunoblotting. Here we present, for the first time, evidences that the protein translation response to insulin is impaired in PW fibroblasts. Insulin alone has a major upregulatory effect on protein kinase B (AKT), glycogen synthase kinase (GSK3beta), while phosphorylation of p70S6K1 protein elongation factor controlled by mammalian target of rapamycin complex I (mTORC1) is reduced. In addition, we provide data that the response to insulin in PW cells can be restored by previous treatment with the amino acid L-Leucine (L-Leu). Our experiments in primary cell cultures demonstrate an impairment of insulin signaling that can be rescued by supplementation with the branched aminoacid L-Leu, indicating a possible therapeutic approach for alleviating muscle mass loss in PW patients.
\end{abstract}

Keywords: Prader Willi Syndrome, protein synthesis, primary fibroblasts, p70-kDa S6 protein kinase, L-Leucine

\section{Introduction}

Prader-Willi (PW) syndrome is a complex neurogenetic disorder caused by the lack of expression of genes located on paternal chromosome 15q11-q13. It is characterized by severe obesity, mild to severe mental retardation and hypotonia. Muscle hypotonia and weakness is present during the neonatal stage and in childhood. It improves with time but adults remain mildly hypotonic with a decreased muscle mass (1). As consequence, patients show an altered motor performance, abnormal gait pattern and overall

Released online in J-STAGE as advance publication December 14, 2015.

*Address correspondence to:

Dr. Elisabetta Fortunati "Mauro Baschirotto" Institute for Rare Diseases - B.I.R.D., 36023 Costozza di Longare, Vicenza. Italy. E-mail: elisabetta.fortunati@birdfoundation.org a decreased physical activity that it is considered to contribute to overweight and frailty. Physical rehabilitation is commonly used to improve skeletal mass performance and to control obesity of adult PW patients. A retrospective analysis of a $\mathrm{PW}$ patient cohort, trained at our institute, showed that intense physical exercise programs have beneficial effects on body weight, but do not improve muscle mass of patients, despite normal calories intake (2). Our data suggested that the regulation of muscle mass was defective in PW patients.

Atrophy is a complex physiological process in which wasting or decreasing in size of tissues or organs undermine their functions. Various cells and tissues, including muscles, undergo atrophy in response to factors such ageing (sarcopenia), disuse, in fasting or disease states such as cancer cachexia, diabetes (3), obesity (4). The extent of cell atrophy is determined, at least in part, by changes in cell capacity of promoting 
cell growth through the modulation of the protein synthesis process. The mammalian target of rapamycin complex 1 (mTORC1) is the key regulator of initiation and elongation of mRNA translation in response to diverse upstream signals including insulin (and other growth factors), amino acids and energy availability (5). The insulin signaling pathway sequentially involves the insulin receptor substrate-1 and -2 (IRS-1 and -2), phosphatidylinositol (PI) 3-kinase, and protein kinase $\mathrm{B}$ (PKB), also known as protein kinase B (AKT), that regulates the $\mathrm{mTORC} 1$ activity and the downstream effectors: the p70-kDa S6 protein kinase (p70S6K1) and the eukaryotic initiation factor 4E-binding protein (4E-BP1) (6). Both proteins modulate translation initiation, by controlling the binding of mRNA to the 40S ribosomal subunit (7).

mTORC1 can also be activated independently by branched-chain amino acids such as L-Leucine (L-Leu), through an inside-out mechanism recently described (8), where leucyl-tRNA synthetase is identified as the intracellular sensor for amino acid signaling to mTORC1 (9). The stimulatory effects of L-Leu on protein synthesis have been recorded in several model systems and it is known to interact with insulin signaling pathway by modulating its phosphorylation events (10). The key role of mTORC1 in muscular trophism has been shown in several mouse models where the specific ablation of mTOR or Rictor induced specifically in the muscle, resulted in progressive dystrophic phenotypes (11). Furthermore, some evidences in humans suggest that mTORC1 and p70S6K1 protein expression (12) or phosphorylation status (13) is reduced in muscles from sarcopenic individuals, underlying the importance of the signaling through mTORC1 in the metabolic regulation of cell atrophy.

At present there are no evidences of the existence of molecular dysfunctions leading to PW cell wasting, or the role of protein translation in PW's muscle loss. Giving the hypothesis that cell atrophy is associated with alterations in protein synthesis, we wanted to verify the protein synthesis activity in response to insulin in PW cells and, if altered, whether stimulation of mTORC1 by L-Leu might rescue the associated defect.

In order to circumvent possible health complications arising from muscle biopsies taken from patients, and in absence of suitable muscle models for our exploratory investigations, we decided to use skin fibroblasts from PW individuals, hoping that the supposed alterations would be maintained in this type of cells.

In this report, we show for the first time, evidences that the protein synthesis response to insulin is impaired in PW primary fibroblasts, and that L-Leu treatment restores the defective response. Our data support the therapeutic potential of the mTORC1 stimulation in Prader Willi disease.

\section{Materials and Methods}

\subsection{Human primary cell cultures}

Human primary skin cells were obtained from 4 adult patients with a clinical diagnosis of PW syndrome established by fluorescent in situ hybridization and DNA methylation-specific PCR assay. All analysed patients showed deletions in chromosome 15q11-q13. Informed written consent was obtained from each subject before participation in the study, after the nature, purpose and risks of the study were explained. All experimental procedures involved in this study were formally approved by the Research Ethics Committee of the Foundation. Primary cell lines used in this study were established starting from sterile, non-necrotic biopsy samples, taken from the forearm of PW patients and directly transported in laboratory facilities in 50 $\mathrm{ml}$ tubes containing culture medium supplemented with antimycotic (fungizone, Sigma-Aldrich, St. Louis, MO, USA) and a double concentration of antibiotics (pen/strep, Sigma-Aldrich, St. Louis, MO, USA). Skin punches were then thoroughly washed with PBS and tissue was mechanical fragmented into 1-2 $\mathrm{mm}$ in size. The fragments were distributed in 24-well plates and grown in Chang culture medium (Irvine scientific CA, USA) containing 30\% foetal bovine serum (FBS, Thermo Fisher Scientific Waltham, Ma, USA), antibiotic-antimycotic solution (1X), L-glutamine (2 $\mathrm{mM}$ ). After $72 \mathrm{~h}$, the extent of tissue adhesion and cell growth was assed using phase-contrast microscopy. Subsequently tissue fragments were removed and primary cells were expanded in Chang medium supplemented with $10 \%$ FBS until freezing. Control fibroblasts were obtained at surgery from the neck or arm skin of 5 control subjects. Biopsies were processed as described above and the primary fibroblasts isolated from skin tissue were used as controls. Primary cells maintained the proliferative capacity with a doubling time of $24 \mathrm{~h}$ for more than 15 passages. Cells between passages 3 and 15 were used for experiments. Cells were routinely tested for Mycoplasma contamination and for senescence by the senescence-associated $\beta$ galactosidase assay (14).

\subsection{Insulin, Serum and L-Leu stimulation experiments}

Human control and PW fibroblasts were seeded one day before at $0.15 \times 10^{6}$ cells in Chang medium containing $10 \%$ FBS. Cells were thoroughly washed with PBS and incubated with $20 \%$ serum containing medium or deprived of serum for $2 \mathrm{~h}$ prior to exposure to $100 \mathrm{nM}$ insulin (Sigma-Aldrich, St. Louis, MO, USA) for 15 min. In the experiments with L-Leu (Sigma-Aldrich, St. Louis, MO, USA), serum deprived cells were preincubated with L-Leu for one hour and then stimulated with $100 \mathrm{nM}$ insulin for additional $15 \mathrm{~min}$. We used 
a $100 \mathrm{nM}$ concentration because it represents a wellaccepted level of insulin stimulation in cell cultures commonly found in the literature (10). At the end of the treatment, cells were washed twice with cold PBS and cells were lysed. Total proteins were extracted as indicated in the immunoblotting paragraph. Each experiment was repeated at least three times including cell cultures, in order to have available biological replicates. Measurements of each biological replicate were repeated in a number of 3 technical replicates, performed on different blots.

\subsection{Immunoblotting}

Cells were lysed with a buffer containing $50 \mathrm{mM}$ Tris- $\mathrm{HCl}, 0.5 \% \mathrm{NP} 40,150 \mathrm{mM} \mathrm{NaCl}, 1 \mathrm{mM}$ EDTA, $1 \mathrm{mM}$ PMSF and proteases inhibitors (Cocktail set II, Calbiochem, Merck, Darmstadt, Germany). After centrifugation at $12000 \mathrm{xg}$ at $4^{\circ} \mathrm{C}$ for 15 min, supernatants were removed and total protein concentration was measured using the QuantiPro ${ }^{\mathrm{TM}}$ BCA Assay (Sigma-Aldrich, St. Louis MO, USA). $40 \mu \mathrm{g}$ of protein homogenates were run on $10 \%$ SDS polyacrylamide gels and transferred onto a polyvinylidene fluoride membrane (PVDF, BioRad Laboratories, Carlsbad, CA, USA) using a semi dry system Trans-blot apparatus. Even transfer of proteins on membranes was evaluated by Ponceau $\mathrm{S}$ (Sigma-Aldrich, St. Louis MO, USA) according to the manufacturing protocol. Membranes were then incubated in Tris-buffered saline $(\mathrm{pH} 7.5)$ containing $5 \%$ BSA and $0.1 \%$ tween-20 (TBST buffer) for $1 \mathrm{~h}$ at room temperature. The following phospho-specific antibodies were added and incubated overnight at $4{ }^{\circ} \mathrm{C}$ in blocking buffer: p70S6K Thr389, GSK3betaSer9 (Cell Signaling Technology, Danvers, MA, USA) and AKTSer473 (Santa Cruz Biotechnology, Inc., Santa Cruz, CA, USA). Membranes were subsequently washed and incubated with anti-rabbit HRP-conjugated secondary antibody (1:10000; EDM Millipore) for $1 \mathrm{~h}$ at room temperature. Following this, membranes were washed repeatedly as before, and immune complexes were visualised on films, (Kodak, Sigma-Aldrich, St. Louis MO, USA) using enhanced chemiluminescence solution (Supersignal West Pico, Pierce, Thermo Fisher Scientific Waltham, Ma, USA). For normalisation, blots were mild stripped and then subsequently reprobed for total p70S6K, total GSK3beta, and AKT proteins to verify the relative amount of analysed proteins. Scanned images of photographic films were produced on a flatbed scanner and the intensity of the bands was quantified using ImageJ open source software (http:// rsb.info.nih.gov/ij/index.html). Images were adjusted in size and brightness, and backgrounds removed by using the "rolling ball" algorithm and transformed to bright background with dark protein bands. Intensity of bands was measured as optical density (O.D.) units by using a calibrated tablet with a density range of 0.05 to 3.05 O.D. In addition, for comparison between different blots, the phosphorylated fraction of kinases was corrected for the total fraction of the protein.

\subsection{Protein synthesis measured by the Surface Sensing of Translation}

A recent developed method, known as surface sensing of translation (SUnSET) allows the measurement of protein synthesis in vitro and in vivo $(15,16)$. This non-radioactive technique involves the use of puromycin, a structural analog of tyrosyl-tRNA, and of an anti-puromycin antibody for the detection of nascent puromycin-labelled peptides by Western blot. Briefly, primary PW fibroblasts and control cells were cultured on 6-well dishes in Chang medium supplemented with $10 \% \mathrm{FBS}$. Upon confluence, cells have been switched to serum free Dulbecco's modified Eagle's medium (DMEM, Thermo Fisher Scientific, Waltham, Ma, USA) for $16 \mathrm{~h}$. The medium was then replaced with fresh medium with or without $100 \mathrm{nM}$ insulin for $60 \mathrm{~min}$. Subsequently, $1 \mu \mathrm{M}$ Puromycin (Merck, Darmstadt, Germany) was added to all wells for an additional $30 \mathrm{~min}$. Cells were then lysed and total proteins were subjected to western blot. For the detection of puromycin-conjugated peptides, PVDF membranes were incubated overnight at $4^{\circ} \mathrm{C}$ with a mouse IgG2a monoclonal anti-puromycin antibody (clone 12D10, 1:5000, Merck, Darmstadt, Germany) dissolved in TBST containing 1\% BSA. After several washes, membranes were incubated for $1 \mathrm{~h}$ at room temperature in 5\% milk-TBST containing horseradish peroxidise conjugated anti-mouse IgG Fc 2 a antibody (1:50,000 Jackson ImmunoReasearch Laboratories Inc., West Grove, PA, USA). The immune complexes were visualized by enhanced chemiluminescence solution. After the capture of relevant images on photographic films the PVDF membranes were stained with Coomassie Blue to verify equal loading of proteins. The rate of protein synthesis was calculated by determining the intensity of each whole lane (incorporating the entire molecular weight range of puromycinlabeled peptides) of the three replicate samples and by correcting for total protein intensity measured on PVDF stained membranes using the ImageJ software. Experiments were performed in triplicates and data are expressed as arbitrary units (a.u.).

\subsection{Statistics}

All analyses were performed by the statistical package SPSS, v.16.0 (SPSS Inc., Chicago, IL, USA). Student $t$-test and one-way analysis of variance assay were used for comparison of untreated and stimulated conditions in control and PW cells. A $p$ value of $p<0.05$ was accepted as the statistically significant. 


\section{Results}

\subsection{PW cells do not increase protein synthesis in response to insulin}

Skin fibroblasts from PW patients were used as cellular model for the verification of possible dysfunctions in the regulation of protein synthesis. Insulin and its related growth factors, has been shown to stimulate protein synthesis in a variety of cells in culture (17). Hence, we measured the insulin capacity of promoting protein synthesis in PW cells, using the non-radioactive SUnSET method. This technique quantifies the puromycin-labeled peptides by immunodetection, as measurement of global protein synthesis (15). Fibroblasts from control and patients were serum deprived for an overnight and stimulated for $1 \mathrm{~h}$ with insulin, followed by $30 \mathrm{~min}$ incubation with puromycin. The translation activity recorded in 5 independent control samples increased significantly after exposure to insulin (5260 a.u. \pm 972 vs. stimulated cells 8205
A

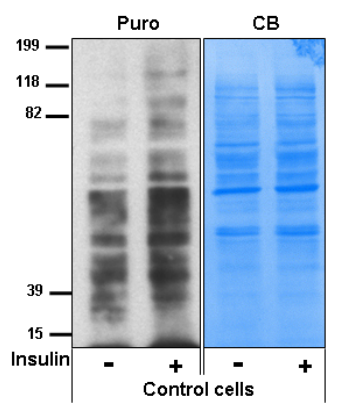

C

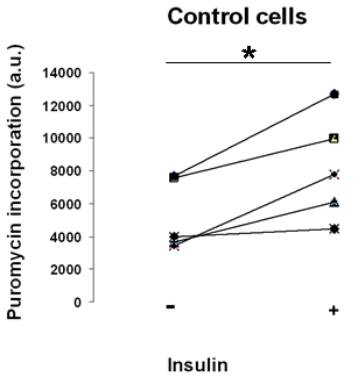

B

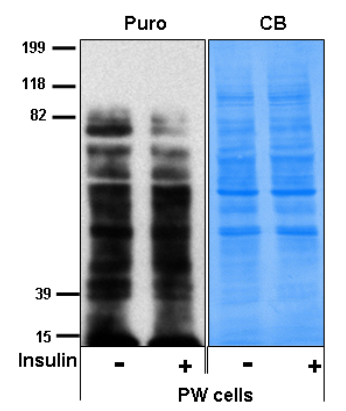

D

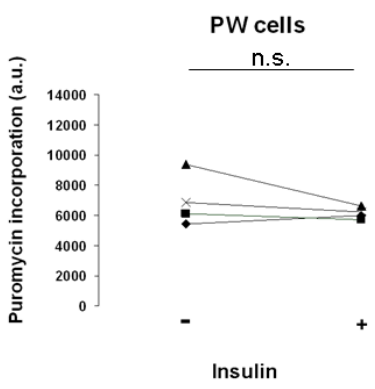

Figure 1. Insulin fails to stimulate protein synthesis in PW cells. A non-radioactive assay (SUnSet test) was used to evaluate protein synthesis in 5 controls and $4 \mathrm{PW}$-derived fibroblasts. Primary cells were serum deprived for $16 \mathrm{~h}$. The medium was then replaced with fresh serum-free DMEM with (+) or without (-) $100 \mathrm{nM}$ insulin for $1 \mathrm{~h}$ and the incorporation of puromycin-labeled peptides was determined as described in materials and methods. (A) and (B): Representative images of WB analysis for puromycin (Puro) - and Coomassie Blue staining (CB) to verify equal loading of proteins - in Controls (A) and PW cells (B). (C) and (D): Quantification of protein translation in response to insulin is expressed as arbitrary units (a.u.) and it is shown in panel (C) (Controls $n=5$ ) and (D) (PW cells $n=4$ ). Each data points are the mean of experiments conducted in triplicates. Data reported are means \pm SEM. The statistical significance level was set at $p<0.05\left(^{*}\right)$; n.s. non significant. a.u. $\pm 193, p<0.05$ ), whereas the rate of puromycin incorporation was unaltered in skin fibroblasts derived from 4 PW individuals (6933 a.u. \pm 862 vs. stimulated cells 6134 a.u. $\pm 193, p=0.43$ ) (Figure $1 \mathrm{C}$ and 1D). We measured no significant difference between PWs and controls in the rate of protein translation at baseline (without serum), although the puromycin incorporation was $25 \%$ higher in patients compared to controls (compare Figure $1 \mathrm{~A}$ and 1B, Puro panels). We conclude that protein synthesis is unresponsive to insulin in PW fibroblasts.

\subsection{PW fibroblasts do not activate p70S6K1 after insulin treatment}

In order to gain insight into the changes of the insulin signaling pathway occurring in PW cells and responsible of the observed reduction of protein synthesis, we monitored the main phosphorylation events on pivotal kinases. Initially, we analyzed the activity of AKT/PKB in consideration of its central role as mediator of insulin signaling (18). Subsequently we focused on the action of active AKT/PKB that promotes initiation of protein translation (17) by phosphorylating the GSK3beta. As last, we considered the effects of AKT/PKB activation on mTORC1, which in turns regulates p70S6K1, leading to an increased protein elongation during protein synthesis. The phosphorylation profile of these 3 kinases was determined after exposure to $20 \%$ serum or to insulin
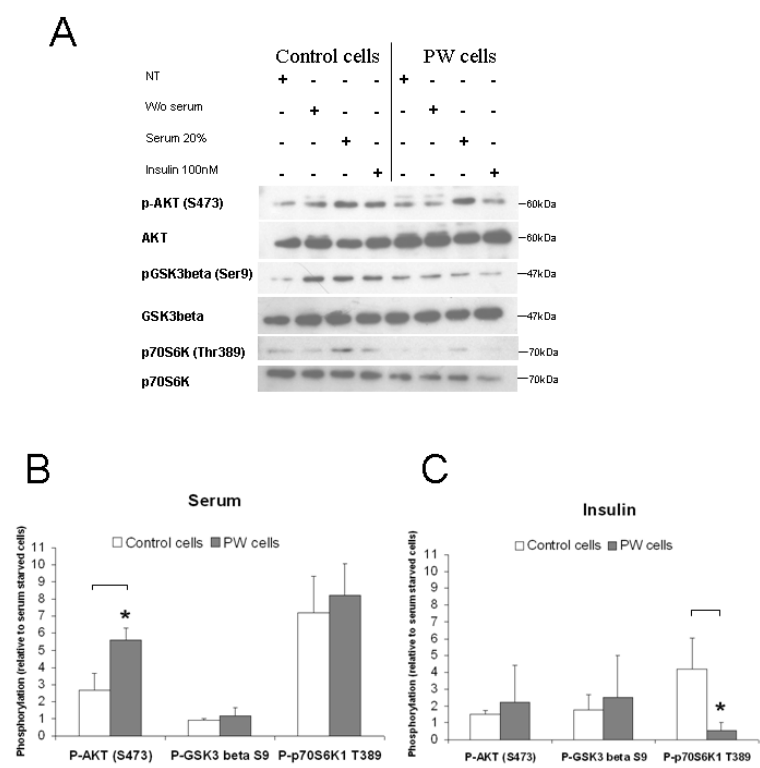

Figure 2. Insulin activates AKT but not p70S6K1. (A): Representative Western blots of PW and control cells for pAKT S473 and total AKT, pGSK3betaS9 and total GSK3beta, p70S6K1T389 and total p70S6K1, after exposure to $20 \%$ serum or to $100 \mathrm{nM}$ Insulin. NT, Not treated; W/o serum, without serum. (B) and (C): Phosphorylation profile obtained by densitometric quantification of the effects of insulin and serum respectively. Data are means $\pm \operatorname{SEM}(n=3)$. The statistical significance level was set at $p<0.05\left({ }^{*}\right)$. Open bars, control cells; solid bar, PW cells. 
A

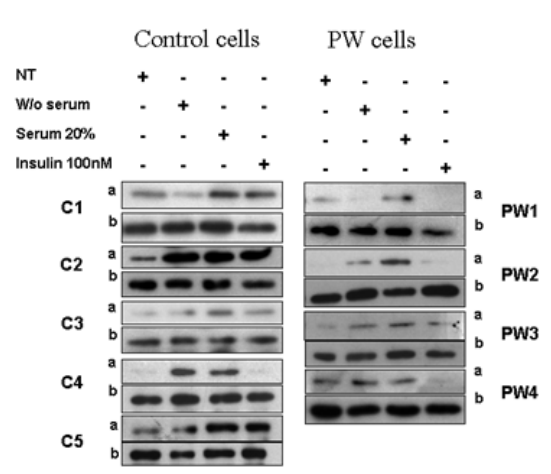

$\mathrm{B}$

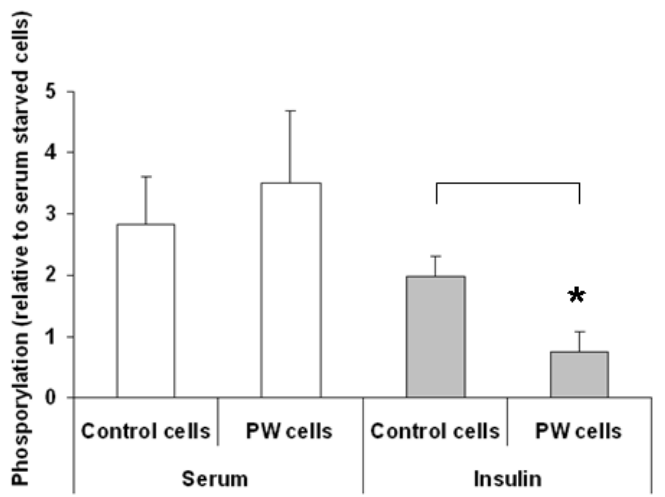

Figure 3. PW cells show low p70S6K1 activation in response to insulin. (A): Representative blots of the expression level of p70S6K1 T389 (a) and total p70S6K1 (b), after treatment with $20 \%$ serum or insulin of controls (C1-5) and patients (PW14). NT, Not treated cells; W/o serum, without serum. (B): Densitometric quantification of the effects of treatments on the analyzed cells. Open bars, serum stimulation; solid bar, Insulin stimulation. Data reported are means \pm SEM. The statistical significance level was set at $p<0.05\left(^{*}\right)$.

(Figure 2). Serum increased the phosphorylation of all 3 kinases, with a significant upregulatory effect on $\mathrm{AKT} / \mathrm{PKB}$ in patient compared to control cells (Figure 2A and 2B). Likewise, insulin treatment induced a phosphorylation increment of AKT/PKB and GSK3beta, although to a less extent compared to serum. A substantial difference was recorded for the phosphorylation of p70S6K1 that was significantly higher in control and PW fibroblasts stimulated with serum while no insulin-mediated increase of p70S6K1 phosphorylation was observed in PW fibroblasts (Figure 2C).

To assess whether the dysfunctional insulin response is a feature of PW fibroblasts, we extended the analysis to 3 additional $\mathrm{PW}$ patients and 4 controls. The AKT/PKB phosphorylation profile resembles that one shown in Figure 2, confirming that insulin activates the AKT/PKB-mediated signaling in both control and patient groups (data not shown). By focusing on signaling downstream of AKT/PKB, we quantified the p70S6K1 phosphorylation in response to stimulations.
A

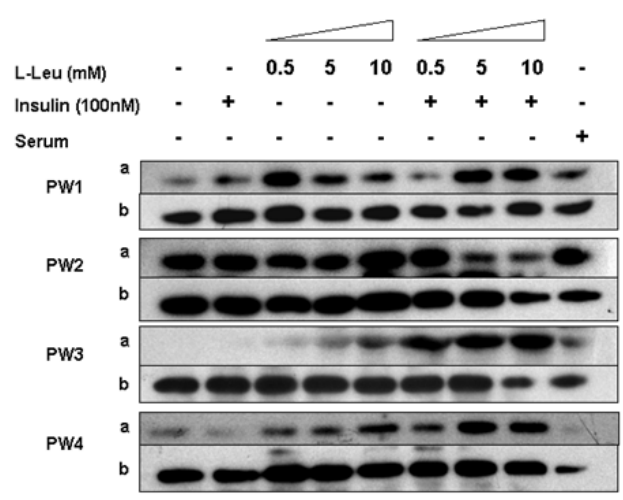

B

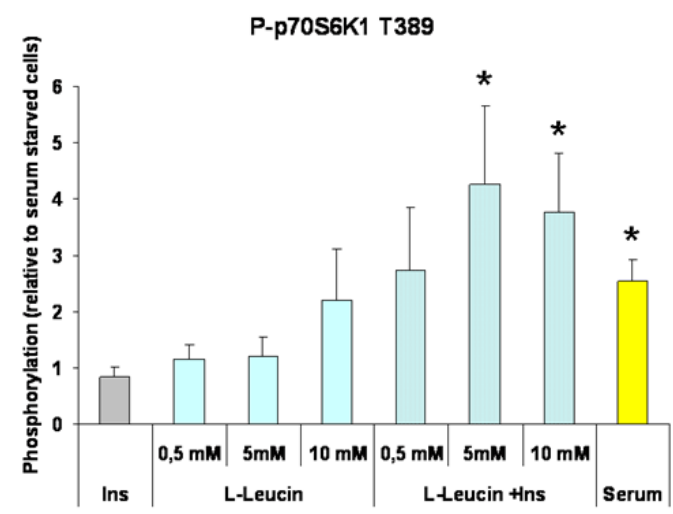

Figure 4. Leucine pretreatment augments insulin effect on p70S6K1 in PW cells. (A): Representative blots of the expression level of p70S6K1 T389 (a) and total p70S6K1 (b), after pre-treatment with L-Leu $(0.5,5,10 \mathrm{mM})$ alone or in combination with insulin (Ins, $100 \mathrm{nM}$ ) in PW cells (PW1-4). $20 \%$ serum stimulation was used as positive control (serum). (B): Densitometric quantification of the effects of stimuli on $\mathrm{PW}$ cells. Data are means \pm SEM. The statistical significance level was set at $p<0.05\left(^{*}\right)$.

All control fibroblasts showed an increment of the p70S6K1 phosphorylation at T389 following exposure to serum and four in five control samples to insulin. On the contrary, none of the four PW patients responded to insulin by increasing the p70S6K1 activation, while all responded to serum (Figure $3 \mathrm{~A}$ and $3 \mathrm{~B}$ ). Overall, our data support the existence in PW cells of a dysfunctional protein translation response to insulin, mediated by AKT/mTORC1/p70S6K1 axis.

\subsection{L-Leu pretreatment augments insulin effect on p70S6K1 signaling}

Protein synthesis is stimulated by the availability of amino acids and energy (ATP). Besides their use as substrate for the building of polypeptide chains, the essential amino acids have been shown to directly activate the regulatory proteins of mRNA translation (19). Particularly interesting is the effect of the branched amino acid L-Leu, which enhances translation initiation and protein synthesis via induction 
of the mTOR pathway $(6,20,21)$ and activation of the downstream target p70S6K1. Therefore L-Leu is considered the main anabolic signal responsible for the increase of protein synthesis.

Since both L-Leu and insulin are known activators of p70S6K1, we wondered whether L-Leu could rescue the insulin-mediated p70S6K1 response in PW fibroblasts. The addition to serum deprived cells of 0.5 , 5 or $10 \mathrm{mM} \mathrm{L}-$ Leu for $1 \mathrm{~h}$ followed by $15 \mathrm{~min}$ insulin stimulation, resulted in a significant activation of p70S6K1 quantified in 2.7-folds at $0.5 \mathrm{mM}, 4.2$-folds at $5 \mathrm{mM}$ and 3.7 -folds at $10 \mathrm{mM}$ (Figure $4 \mathrm{~A}$ and $4 \mathrm{~B}$ ). No significant effects on the kinase activity of L-Leu alone were detected, despite a slight improvement observed at $5 \mathrm{mM}$ (1.2-folds) and at $10 \mathrm{mM}$ (2.2-folds) L-Leu. These results suggest beneficial effects of L-Leu administration to PW cells and confirmed the role of L-Leu as modulator of the insulin signaling pathway and in particular of the protein translation via mTORC1.

\section{Discussion}

The regulation of protein synthesis by specific metabolic signaling pathways is crucial for normal cell growth. In a patient cohort trained at our institute we observed that muscular mass did not increase after training in a normal dietary regimen (2). Muscle mass loss in PW patients has been pointed out by others $(1,22)$ and contributes to alterations in motor performance that leads to obesity and severe disabilities. The causes of muscle weakness, and motor problems in PW patients are not clear. In this paper we explored the possibility that muscle mass loss at cellular level may be caused by alteration in protein translation, impairing cellular growth. Our hypothesis was tested on skin primary fibroblasts of control and PW group exposed to growth stimuli. As mentioned earlier, the choice of this model was determined by the absence of a suitable in vitro model for PW muscle, and by the risks associated with muscular biopsies for patients. Although we are aware of the limitations of this model, our data may represent a useful step forward in the comprehension of muscle atrophy in PW disease.

Our major finding is that the translation response to insulin is impaired in the PW group compared to controls (Figure 1). The baseline of translational activity in absence of serum was overall higher in diseased cells (Figure 1A and 1B, Puro panel), suggesting that their metabolic phenotype could be slightly more active compared to controls. Additionally, we recorded more variable response to insulin in control cells as evidenced by the lack of p70S6K1 activation in one of the control sample (Figure 3A, Control cells, C4a, Insulin $100 \mathrm{nM})$.. It may be possible that the variability of control group selected on the absence of apparent diseases and availability of biopsies, mirrors underestimated biological factors in few samples.
Nevertheless, we considered more appropriate to include all control samples in the statistical analysis. Overall our data showed a dysfunctional translation response in PW patients.

The analysis of the phosphorylation profile induced by serum showed no changes in the activation in diseased or control cells. On the contrary, the molecular analysis of the insulin mediated signaling pathway showed that while AKT/PKB and GSK3beta activity is unchanged (Figure 2), the phosphorylation of p70S6K1 is decreased in PW cells (Figure 2 and Figure 3). Since the AKT activity, as well as the downstream target GSK3beta, is comparable in PW and control fibroblasts, our data suggest that the dysfunction to insulin response resides in mTORC1/p70S6K1 axis. A further support to the existence of an alteration in the mTORC $1 / \mathrm{p} 70 \mathrm{~S} 6 \mathrm{~K} 1$ signaling pathway is the rescue effect exerted by L-Leu treatment on Insulin mediated phosphorylation of p70S6K1 recorded in PW fibroblasts (Figure 4).

It is known that L-Leu is a modulator of the insulin activity in several in vitro and in vivo models $(10,23)$ by regulating the mTORC 1 activity. The mechanism by which L-Leu activates mTORC1 has been recently elucidated (8). According to this model, L-Leu stimulates mTORC1 activity in a manner that requires v-ATPase, the Ragulator and the Reg GTPase, and favours the translocation of mTORC1 to the membrane compartment of the lysosomes, where Rheb (small GTPase Ras homolog enriched in brain), phosphorylates mTORC1 activating it (Figure 5).

In our experimental settings, L-Leu alone shows slight effects of p70S6K1 phosphorylation, indicating that mTORC1 is not fully active (Figure 4). Since the magnitude and rate at which mTORC1 is activated by L-Leu is reduced in KO cells for Reg GTPase (8), it is tempting to speculate that dysregulation of RegGTPase, which binds to and recruits mTORC1 to lysosomes, might be the possible cause of the modest effects of L-Leu treatment.

Addition of insulin to L-Leu treated cells resulted in a significant enhancement of the p70S6K1 phosphorylation levels and therefore of mTORC1 activity (Figure 4). Insulin activates mTORC1 through AKT/PKB mediated phosphorylation of PRAS40 and TSC1/TSC2 complex, preventing their inhibitory effects on mTORC1 $(24,25)$ and favours the docking of mTORC1 to lysosome where mTORC1 is activated by Rheb (Figure 5). It is conceivable that alterations of mTORC1 activation observed in PW fibroblasts are the result of pathological modifications in the interaction between mTORC1 and regulatory proteins, Rags, Rheb and PRAS40, induced by L-Leu or insulin.

Given the complexity of the mTORC1 pathway and the presence of feedback loops between components, further investigations are needed to establish the mechanisms underlying our observations. In addition we cannot exclude that other pathways in addition to 


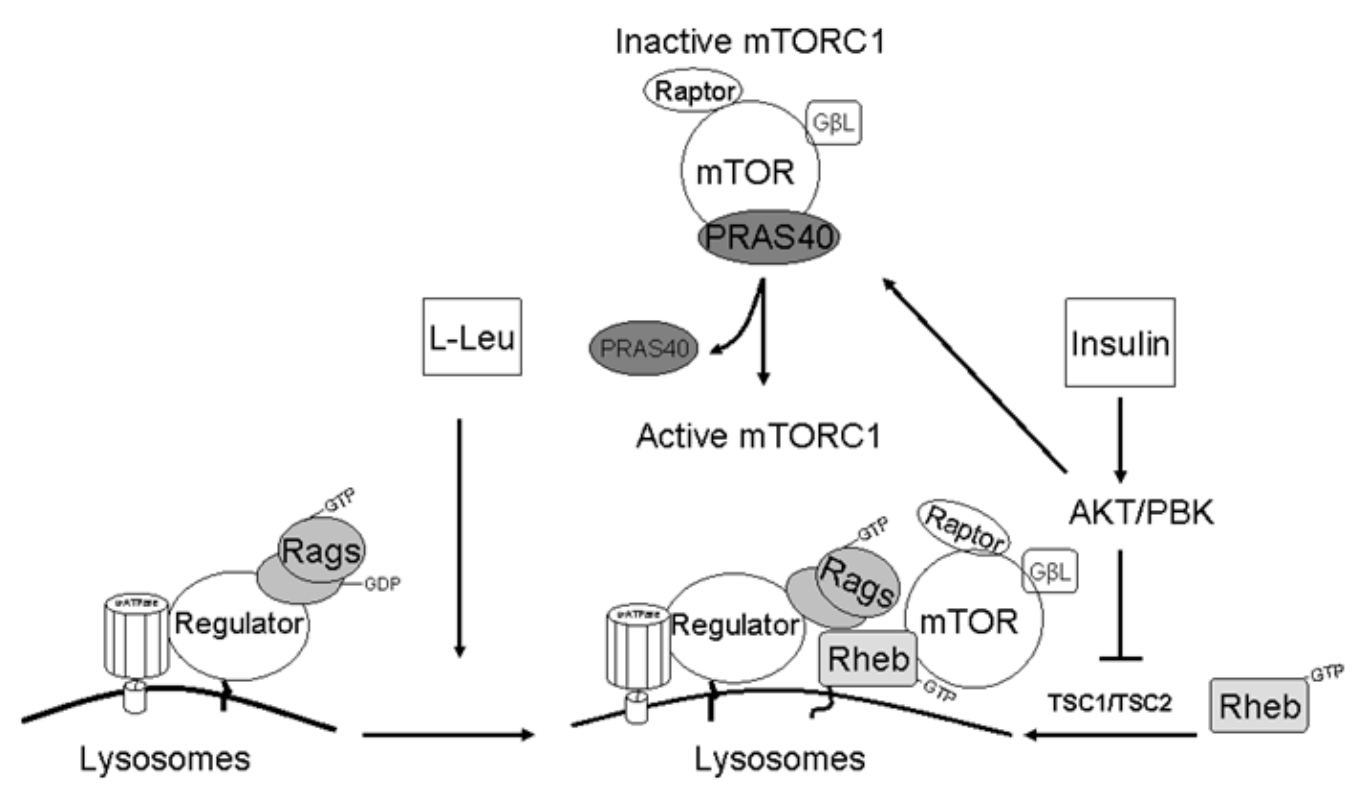

Figure 5. Critical regulatory proteins in leucin and insulin signaling to $\mathbf{m T O R C 1}$. Protein translation is controlled by the activity of mTOR. L-Leu or insulin activates mTOR through Rag GTPase or Rheb GTPase, respectively. Insulin stimulation after pre-treatment with L-Leu favours the modulation of regulatory proteins such as raptor, PRAS40, Rheb, and the Rag GTPases that leads to increase protein stimulation in PW cells. Critical proteins are filled in grey. mTORC1 is composed of the serine/threonine protein kinase, mTOR, the regulatory associated protein of TOR, raptor, and the G-protein $\beta$-subunit like protein, G $\beta$ L. PRAS40 (proline-rich Akt substrate of 40-kDa); TSC1/TSC2 (tuberous sclerosis complex 1 and 2); RagsGTPase (small guanosine triphosphatases).

mTORC1/p70S6k1 may play a role in the regulation of protein translation in response to growth factors. In this context p70S6K1 has emerged as a critical signaling component in the development of insulin resistance through phosphorylation and inhibition of insulin Receptor 1 and 2 functions (26).

The evidences reported in this manuscript were obtained from a limited number of patients, but they represent a good starting point for further investigations. Our data suggest a common dysfunction in the regulation mechanism of protein translation in response to insulin in PW fibroblasts. In addition, our in vitro data show a possible molecular link between alterations in protein synthesis in vitro and reduced muscle mass growth observed in vivo in PW patients (23).

We are aware that the results obtained with patient fibroblasts, cannot reproduce the complexities of what occurs in muscle cells in vivo. Therefore our findings must be further evaluated in different models such as patient muscle biopsies or PW reprogrammed fibroblasts differentiated into muscle cells. Nevertheless, patientderived skin fibroblasts have been proven to be suitable disease models for studying phosphorylation events in response to growth factors because of their dependency to trophic signals. PW primary fibroblasts therefore must be regarded as a useful in vitro model for studying signal transduction.

In conclusion, in this study we showed for the first time that protein translation in response to insulin is dysfunctional in PW primary fibroblasts and it is associated with a decrease in P70S6K1 phosphorylation. Since protein synthesis regulation is crucial for maintenance of cellular growth, our data support a molecular basis for the motor problems observed in PW patients. Finally we show that preincubation with the branched amino acid L-Leu can restore the insulin sensitivity suggesting its possible use in the treatment of muscle mass loss of PW patients.

\section{Acknowledgements}

This work was supported by a research grant from the Canadian Foundation for Prader Willi Research (FPWR) in 2013-2014 and co-founded by the Mauro Baschirotto Foundation for Rare Diseases.

\section{References}

1. Cassidy SB, Schwartz S, Miller JL, Driscoll DJ. PraderWilli syndrome. Genet Med. 2011; 14:10-26.

2. Grolla E, Andrighetto G, Parmigiani P, Hladnik U, Ferrari G, Bernardelle R, Lago MD, Albarello A, Baschirotto G, Filippi G, Lovato R, Dolcetta D. Specific treatment of Prader-Willi syndrome through cyclical rehabilitation programmes. Disabil Rehabil. 2011; 33:1837-1847.

3. Krause MP, Riddell MC, Hawke TJ. Effects of type 1 diabetes mellitus on skeletal muscle: Clinical observations and physiological mechanisms. Pediatr Diabetes. 2011; 12:345-364.

4. Akhmedov D, Berdeaux R. The effects of obesity on skeletal muscle regeneration. Front Physiol. 2013; 4:371.

5. Laplante M, Sabatini DM. mTOR signaling in growth control and disease. Cell. 2012; 149:274-293.

6. Kimball SR, Shantz LM, Horetsky RL, Jefferson LS. Leucine regulates translation of specific mRNAs in 
L6 myoblasts through mTOR-mediated changes in availability of eIF4E and phosphorylation of ribosomal protein S6. J Biol Chem. 1999; 274:11647-11652.

7. Kimball SR, Farrell PA, Jefferson LS. Invited review: Role of insulin in translational control of protein synthesis in skeletal muscle by amino acids or exercise. J Appl Physiol (1985). 2002; 93:1168-1180.

8. Jewell JL, Kim YC, Russell RC, Yu FX, Park HW, Plouffe SW, Tagliabracci VS, Guan KL. Metabolism. Differential regulation of mTORC1 by leucine and glutamine. Science. 2015; 347:194-198.

9. Han JM, Jeong SJ, Park MC, Kim G, Kwon NH, Kim HK, Ha SH, Ryu SH, Kim S. Leucyl-tRNA synthetase is an intracellular leucine sensor for the mTORC1signaling pathway. Cell. 2012; 149:410-424.

10. Di Camillo B, Eduati F, Nair SK, Avogaro A, Toffolo GM. Leucine modulates dynamic phosphorylation events in insulin signaling pathway and enhances insulindependent glycogen synthesis in human skeletal muscle cells. BMC Cell Biol. 2014; 15:9.

11. Risson V, Mazelin L, Roceri M, et al. Muscle inactivation of mTOR causes metabolic and dystrophin defects leading to severe myopathy. J Cell Biol. 2009; 187:859-874.

12. Cuthbertson D, Smith K, Babraj J, Leese G, Waddell T, Atherton P, Wackerhage H, Taylor PM, Rennie MJ. Anabolic signaling deficits underlie amino acid resistance of wasting, aging muscle. FASEB J. 2005; 19:422-424.

13. Li M, Verdijk LB, Sakamoto K, Ely B, van Loon LJ, Musi N. Reduced AMPK-ACC and mTOR signaling in muscle from older men, and effect of resistance exercise. Mech Ageing Dev. 2012; 133:655-664.

14. Itahana K, Itahana Y, Dimri GP. Colorimetric detection of senescence-associated beta galactosidase. Methods Mol Biol. 2013; 965:143-156.

15. Schmidt EK, Clavarino G, Ceppi M, Pierre P. SUnSET, a nonradioactive method to monitor protein synthesis. Nat Methods. 2009; 6:275-277.

16. Goodman CA, Mabrey DM, Frey JW, Miu MH, Schmidt EK, Pierre P, Hornberger TA. Novel insights into the regulation of skeletal muscle protein synthesis as revealed by a new nonradioactive in vivo technique.
FASEB J. 2011; 25:1028-1039.

17. Proud CG. Regulation of protein synthesis by insulin. Biochem Soc Trans. 2006; 34:213-216.

18. Lizcano JM, Alessi DR. The insulin signalling pathway. Curr Biol. 2002; 12:R236-R238.

19. Norton LE, Layman DK. Leucine regulates translation initiation of protein synthesis in skeletal muscle after exercise. J Nutr. 2006; 136:533S-537S.

20. Peyrollier K, Hajduch E, Blair AS, Hyde R, Hundal HS. L-leucine availability regulates phosphatidylinositol 3-kinase, p70 S6 kinase and glycogen synthase kinase-3 activity in L6 muscle cells: Evidence for the involvement of the mammalian target of rapamycin (mTOR) pathway in the L-leucine-induced up-regulation of system A amino acid transport. Biochem J. 2000; 350:361-368.

21. Gran P, Cameron-Smith D. The actions of exogenous leucine on mTOR signalling and amino acid transporters in human myotubes. BMC Physiol. 2011; 11:10.

22. Reus L, Zwarts M, van Vlimmeren LA, Willemsen MA, Otten BJ, Nijhuis-van der Sanden MW. Motor problems in prader-willi syndrome: A systematic review on body composition and neuromuscular functioning. Neurosci Biobehav Rev. 2011; 35:956-969.

23. Zhang Y, Guo K, LeBlanc RE, Loh D, Schwartz GJ, $\mathrm{Yu} \mathrm{YH}$. Increasing dietary leucine intake reduces dietinduced obesity and improves glucose and cholesterol metabolism in mice via multimechanisms. Diabetes. 2007; 56:1647-1654.

24. Sancak Y, Thoreen CC, Peterson TR, Lindquist RA, Kang SA, Spooner E, Carr SA, Sabatini DM. PRAS40 is an insulin-regulated inhibitor of the $\mathrm{mTORC1}$ protein kinase. Mol Cell. 2007; 25:903-915.

25. Vander Haar E, Lee SI, Bandhakavi S, Griffin TJ, Kim DH. Insulin signalling to mTOR mediated by the Akt/ PKB substrate PRAS40. Nat Cell Biol. 2007; 9:316-323.

26. Tremblay F, Brûlé S, Hee Um S, Li Y, Masuda K, Roden M, Sun XJ, Krebs M, Polakiewicz RD, Thomas G, Marette A. Identification of IRS-1 ser-1101 as a target of S6K1 in nutrient- and obesity-induced insulin resistance. Proc Natl Acad Sci U S A. 2007; 104:14056-14061.

(Received October 22, 2015; Accepted November 30, 2015) 